\title{
A Mathematical Formalism for the Evaluation of C-space for Redundant Robots
}

\author{
Roberto Therón, Vidal Moreno, Belén Curto, and Francisco J. Blanco \\ Departamento de Informática y Automática \\ Facultad de Ciencias - Universidad de Salamanca \\ Plaza de la Merced s/n 37008. Salamanca (Spain) \\ theron@usal.es, vmoreno@abedul.usal.es \\ bcurto@abedul.usal.es, jblanco@abedul.usal.es
}

\begin{abstract}
This paper presents a new general method for obstacle representation in the configuration space (C-space) for redundant robots. The method is based on the analytical deconstruction of the C-space, i.e., the separated evaluation of the C-space portion contributed by the collisions of each link in the kinematic chain. The systematic application of a simple convolution of two functions describing each link and the workspace, respectively, is applied. In order to do that, the transformation of the workspace among reference systems located at one point of each link is needed; in this step a well-known and sound method is used.
\end{abstract}

\section{Introduction}

In this paper the explicit representation of obstacles in the Configuration Space (C-space) of redundant articulated robots is adressed. This representation is widely used in robotics in many tasks that involve the obstacle-avoidance problem as for example path planning or the optimization of robot paths.

The C-space concept [1] is very useful since the robot is representated by a single point at this manifold. The advent of new methods that enable simpler and faster evaluations is a challenge. Consequently, Kavraki [2] suggested the use of the convolution of the obstacles and a mobile robot and the application of the Convolution Theorem to evaluate the discrete C-space. Although, it is doubtless a great advance on the way to optimize the computation time for the C-space evaluation, only 2D mobile robots were considered, thus lacking in generality.

While a lot of effort has been done dealing with mobile robots, only a few works [3][4][5][6] are concerned with articulated manipulators. In [7] a general method is proposed, for many types of structures that include both mobile and articulated, where the C-obstacles evaluation is established as the convolution product of two functions that represent the robot and the obstacles.

Although this is a big step, the method is not general enough, since some structures - such as redundant robots - can not be considered. A further step can be taken by an analysis of the proposed general method as applied to articulated robots, since changing the reference system for each link would provide a new method that simplifies computation, thus reducing both the memory needs and the computation times [8]. 


\section{Evaluating C-Obstacles as a Convolution}

In this section, the method proposed by Curto in [7] is revised, as it is the basis for the method presented in this paper.

The representation of the C-obstacles is proposed based on the integral of the product of two functions: one that represents the robot $A$ and another one that represents the obstacles in the workspace, $B . W$ will designate the workspace and $C$ the $\mathrm{C}$-space. Thus,

Definition 1. Let $A: C \times W \rightarrow R$ be the function defined by

$$
A(q, x)= \begin{cases}1 & \text { if } x \in \mathbf{A}(q) \\ 0 & \text { if } x \notin \mathbf{A}(q)\end{cases}
$$

where $\mathbf{A}(q)$ is the subset of $W$ that represents the robot at the configuration $q$.

Definition 2. Let $B: W \rightarrow R$ be the function defined by

$$
B(x)= \begin{cases}1 & \text { if } x \in \mathbf{B} \\ 0 & \text { if } x \notin \mathbf{B}\end{cases}
$$

where $\mathbf{B}$ is the subset of $W$ formed by the obstacles.

Using both $A$ and $B$, a new definition for calculating C-obstacles is proposed:

Definition 3. Let $C B: C \rightarrow R$ be the function defined by

$$
C B(q)=\int A(q, x) B(x) d x \quad \forall q \in C, \quad \forall x \in W
$$

The region $\mathbf{C B}_{\mathbf{f}}$ is defined as the subset of $C$ that verifies

$$
\mathrm{CB}_{\mathbf{f}}=\{q \in C / C B(q)>0\}
$$

The previous expressions were defined without considering any specific parameterization of $W$ and $C$.

Now, a representation of $W$ and $C$ is given by selecting two frames $F_{W}$ and $F_{A}$ for the workspace and for the robot, respectively, where $F_{W}$ is fixed and $F_{A}$ is attached to the robot. In this way, a point $x \in W$ is given by $\left(x_{1}, x_{2}, \cdots, x_{n}\right)$ where $n$ is the workspace dimension, and a configuration $q \in C$ is represented by $\left(q_{1}, q_{2}, \cdots, q_{m}\right)$ that specify the position and orientation of $F_{A}$ respect to $F_{W}$, where $m$ is the dimension of $C$. Thus, the expression (3) becomes

$$
C B\left(q_{1}, \cdots, q_{m}\right)=\int A\left(q_{1}, \cdots, q_{m}, x_{1}, \cdots, x_{n}\right) B\left(x_{1}, \cdots, x_{n}\right) d x_{1} \cdots d x_{n}
$$




\section{Superposition Principle of C-obstacles}

In this paper, an articulated robot is considered as a kinematic chain. In this way, a robot $\mathbf{A}$ is viewed as a set of $r$ rigid objects. The kinematics of this chain, i.e., the movement restrictions imposed by the joint to each element, $\mathbf{A}_{i}$ - the degrees of freedom, DOFs-, would determine some regions of the C-space.

This principle is the basis of the evaluation of the C-space for robots that consist of several elements connected by means of different types of joints.

Considering that a robot consists of $r$ rigid objects, the resulting C-obstacles will follow the Superposition Principle:

Theorem 1. Let $\mathbf{A}$ be an articulated robot formed by $r$ elements $\mathbf{A}_{1}, \ldots, \mathbf{A}_{r}$. If $\mathbf{C B}_{1}, \ldots, \mathbf{C B}_{r}$ are, respectively, the $C$-obstacle regions for the $\mathbf{A}_{1}, \ldots, \mathbf{A}_{r}$ elements in the space where the obstacle $\mathbf{B}$ is projected, then, the $C$-obstacle $\mathbf{C B}$ due to $\mathbf{B}$ for the robot $\mathbf{A}$ can be obtained as

$$
\mathbf{C B}=\bigcup_{k=1}^{r} \mathbf{C B}_{k}
$$

The expression (6) reflects the fact that the union of these subsets equals the configuration space for $\mathbf{A}$. The idea of C-obstacles superposition is the key principle that enables the deconstruction approach.

\section{The Deconstruction Method}

The Deconstruction method tries to independently evaluate portions of the Cspace in order to find the C-obstacles due to each link in the kinematic chain.

\subsection{Applicating the Superposition Principle}

Taking into account (6), the calculation of $\mathbf{C B}$ for a robot $\mathbf{A}$, a kinematic chain of $r$ links, is done through the union of all the $\mathbf{C B}_{k}$ related to each of the elements of the robot. The computation of every C-obstacle region must be done through the evaluation of the associated $C B_{k}$ functions.

$$
C B_{k}\left(q_{1_{k}}, \cdots, q_{s_{k}}\right), \forall k \in\{1, \ldots, r\}
$$

with $\left\{q_{1_{k}}, \cdots, q_{s_{k}}\right\} \subseteq\left\{q_{1}, \cdots, q_{m}\right\}$, where $\left\{q_{1}, \cdots, q_{m}\right\}$ are the DOFs associated to the robot $\mathbf{A}$. That is, for the $k$-th element only the subset of configuration variables associated to it are considered, and, analogously to (5), each of the $C B_{k}\left(q_{1_{k}}, \cdots, q_{s_{k}}\right)$ functions is evaluated as follows

$$
\int A_{k}\left(q_{1_{k}}, \cdots, q_{s_{k}}, x_{1}, \cdots, x_{n}\right) B\left(x_{1}, \cdots, x_{n}\right) d x_{1} \cdots d x_{n}
$$




\subsection{Choosing the Frames}

When solving the integral (8), the function $A_{k}\left(q_{1_{k}}, \cdots, q_{s_{k}}, x_{1}, \cdots, x_{n}\right)$, representing the robot, is difficult to evaluate, due to its dependency on all of the DOFs related to itself and to the previous links in the chain. Thus, we will try to reduce this difficulty by choosing the proper frames.

In order to do that, let's consider the robot formed by the kinematic chain of figure 1. As one can see, following the Denavit-Hartenberg method [9], a frame is associated with each link, placing the origin at the end of the link; the orientation of axes depends on the position and orientation of the link.

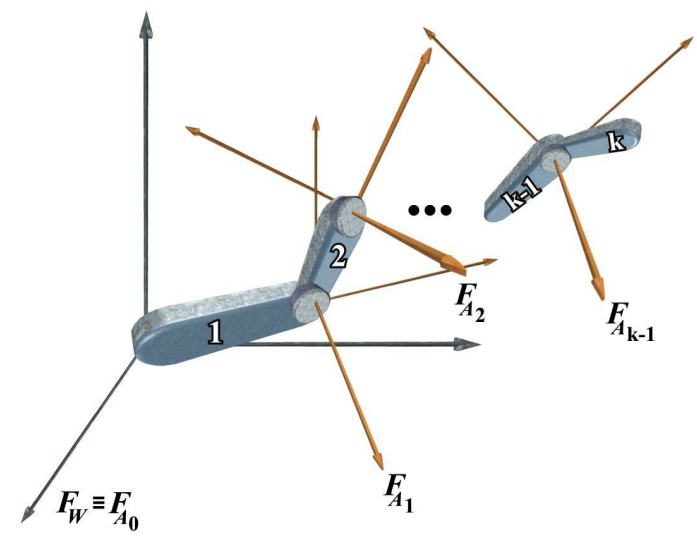

Fig. 1. Frames in the kinematic chain of an articulated robot

Following the Denavit-Hartenberg procedure, the Deconstruction method proposes to use the frame determined by the previous link for the $k$-th element. Thus, for link 1 the frame $F_{A_{0}}$-which coincides with the workspace frame, $F_{W}$ - is used; similarly, for the $k$-th link, frame $F_{A_{k-1}}$ will be used (figure 1).

Now, if we have a look to $A_{k}\left(q_{1_{k}}, \cdots, q_{s_{k}}, x_{1}, \cdots, x_{n}\right)$, the expression we are evaluating, it can be written as follows

$$
A_{k}(\underbrace{q_{1_{k}}, \cdots, q_{u_{k}}}_{D O F_{(1, \ldots, k-1)}}, \underbrace{q_{(u+1)_{k}}, \cdots, q_{s_{k}}}_{D O F_{k}}, x_{1}, \cdots, x_{n})
$$

where $\left\{q_{1_{k}}, \cdots, q_{u_{k}}\right\}$ are the degrees of freedom associated to the elements preceding the k-th element the $k$-th element, whose DOFs are $\left\{q_{(u+1)_{k}}, \cdots, q_{s_{k}}\right\}$.

At this point, the position and orientation of the element $\mathbf{A}_{k}$ is expressed related to the frame $F_{A_{0}}$. The position, just like the frame $F_{A_{k-1}}$, is determined by the associated degrees of freedom of the previous links in the chain, that is to say, some of the parameters related to each $\mathbf{A}_{i}$-previous elements- in that subchain, $\left(a_{i}, \alpha_{i}, d_{i}\right.$ and $\theta_{i}$, the Denavit-Hartenberg parameters). 
Thus, if the position and orientation of the element $\mathbf{A}_{k}$ are expressed taking as origin the frame $F_{A_{k-1}}$, its evaluation will be much simpler. An homogeneous transformation $\mathbf{T}$ is needed to perform this operation.

Definition 4. Let ${ }_{0}^{k-1} \mathbf{T}$ be the transformation that permits us to move the frame $F_{A_{0}}$ to such point that it will coincide with $F_{A_{k-1}}$.

It is important to point out that this homogeneous transformation depends on the configuration parameters related to the previous elements in the chain, that is to say, ${ }_{0}^{k-1} \mathbf{T}=f\left(q_{1_{k}}, \cdots, q_{u_{k}}\right)$. At this point, the position and orientation of the link $\mathbf{A}_{k}$, expressed related to the frame $F_{A_{k-1}}$, will only depend of its associated degrees of freedom, that is, $\left\{q_{(u+1)_{k}}, \cdots, q_{s_{k}}\right\}$.

However, this homogeneous transformation has a consequence: it will be necessary to express the workspace as a function of the new frame, $F_{A_{k-1}}$ :

$$
B^{\prime}\left(x_{1}^{\prime}, \cdots, x_{n}^{\prime}\right)={ }_{0}^{k-1} \mathbf{T} B\left(x_{1}, \cdots, x_{n}\right)
$$

In this way, the evaluation of (9) is equivalent to the following one

$$
A_{k}^{\prime}\left(q_{(u+1)_{k}}, \cdots, q_{s_{k}}, x_{1}^{\prime}, \cdots, x_{n}^{\prime}\right)
$$

Finally, (8), which is used to calculate the C-obstacle portion pertaining to the element $\mathbf{A}_{k}$, becomes

$$
\int A_{k}^{\prime}\left(q_{(u+1)_{k}}, \cdots, q_{s_{k}}, x_{1}^{\prime}, \cdots, x_{n}^{\prime}\right) B^{\prime}\left(x_{1}^{\prime}, \cdots, x_{n}^{\prime}\right) d x_{1}^{\prime} \cdots d x_{n}^{\prime}
$$

Now, after the proper frame is chosen, as it can be seen in (12), it is possible to study individually each one of the links.

\subsection{Choosing the Coordinate Functions}

Kavraki [2] and Curto [7] propose the simplification of the C-space calculation by the use of the Convolution theorem (and the Fast Fourieer Transform). We shall now expose how this is applicable inside the new proposed formalism by means of the introduction of a coordinate functions change.

As demonstrated in [7], it is sufficient to choose the proper coordinate functions, $\left(\xi_{1}, \cdots, \xi_{n}\right)$, that will permit to find one or more relationships between some of the configuration variables and some of the coordinate functions, which will allow to find the convolution.

Thus, a new function, $\bar{A}_{k}^{\prime}$, is introduced; the idea is to find a simpler functional dependency in function $A_{k}^{\prime}$, in such a way that element $\mathbf{A}_{k}$ becomes independent of a subset of $\left\{q_{(u+1)_{k}}, \cdots, q_{s_{k}}\right\}$, depending only on $\left\{q_{(v+1)_{k}}, \cdots, q_{s_{k}}\right\}$.

Having this new function $\bar{A}_{k}^{\prime}$, (12) will be defined as

$$
\int \operatorname{long} A \operatorname{long} B d \xi_{1} \cdots d \xi_{n}
$$




$$
\begin{aligned}
& \operatorname{long} A=\bar{A}_{k}^{\prime}\left(0, \cdots, 0, q_{(v+1)_{k}}, \cdots, q_{s_{k}}, \xi_{1}-q_{(u+1)_{k}}, \cdots, \xi_{v}-q_{v_{k}}, \xi_{(v+1)_{k}}, \cdots, \xi_{n}\right) \\
& \text { long } B=B^{\prime}\left(\xi_{1}, \cdots, \xi_{n}\right)
\end{aligned}
$$

which leads to a function $\bar{A}_{k}^{\prime}$ that depends only on $\left\{q_{(v+1)_{k}}, \cdots, q_{s_{k}}\right\}$. Now, for variables $\left\{q_{(u+1)_{k}}, \cdots, q_{v_{k}}\right\}$ the following convolution product appears.

$$
\int\left(\bar{A}_{\left.k_{\left(0, \cdots, 0, q_{(v+1)_{k}}^{\prime}\right.}^{\prime}, \cdots, q_{s_{k}}\right)} * B\right)_{\left(\xi_{1}, \cdots, \xi_{v_{k}}\right)}\left(\xi_{(v+1)_{k}}, \cdots, \xi_{n}\right) d \xi_{(v+1)_{k}} \cdots d \xi_{n}
$$

where subindices $\left(\xi_{1}, \cdots, \xi_{v_{k}}\right)$ denote that the convolution product is calculated for all of the values of these variables.

\section{Conclusion}

In this paper, a mathematical formalism for the Deconstruction method is proposed. This approach permits the simplification of the C-space evaluating process by means of the application of a simple and repetitive operation for each link in the kinematic chain, being valid for redundant robots. This method can naturally face the evaluation of C-spaces of many dimensions, since only non-colliding configurations are considered for the evaluation of the following links.

\section{References}

1. Lozano-Pérez, T.: Spatial planning: A configuration space approach. IEEE Transactions on Computers 32 (1983) 108-120

2. Kavraki, L.E.: Computation of configuration space obstacles using the fast fourier transform. IEEE Tr. on Robotics and Automation 11 (1995) 408-413

3. Maciejewski, A.A., Fox, J.J.: Path planning and the topology of configuration space. IEEE Tr. on Robotics and Automation 9 (1993)

4. Newman, W., Branicky, M.: Real-time configuration space transforms for obstacle avoidance. The International Journal of Robotics Research 6 (1991)

5. Lozano-Pérez, T.: A simple motion-planning algorithm for general robot manipulators. IEEE Journal of Robotics and Automation 3 (1987) 224-238

6. Lozano-Pérez, T., P.O'Donnell: Parallel robot motion planning. In: Proc. of the IEEE Int. Conf. on Robotics and Automation. (1991) 1000-1007

7. Curto, B., Moreno, V., Blanco, F.J.: A general method for c-space evaluation and its application to articulated robots. IEEE Transactions on Robotics and Automation 18 (2002) 24-31

8. Therón, R., Moreno, V., Curto, B., Blanco, F.J.: Assessing methods for the evaluation of the configuration space for planar revolute manipulators. Journal of Computational Methods in Science and Engineering 4 (2004) 149-156

9. Denavit, J., Hartenberg, R.S.: A kinematic notation for lower-pair mechanisms on matrices. Journal of Applied Mathematics (1955) 215-221 